\title{
Nanobodies as modulators of inflammation: potential applications for acute brain injury
}

\author{
Björn Rissiek ${ }^{1}{ }^{*}$, Friedrich Koch-Nolte ${ }^{2}$ and Tim Magnus ${ }^{1}$ \\ 1 Department of Neurology, University Medical Center Hamburg-Eppendorf, Hamburg, Germany \\ ${ }^{2}$ Department of Immunology, University Medical Center Hamburg-Eppendorf, Hamburg, Germany
}

\author{
Edited by: \\ Christoph Kleinschnitz, University of \\ Würzburg, Germany \\ Reviewed by: \\ Anna M. Planas, Consejo Superior \\ de Investigaciones Científicas \\ (IIBB-CSIC) and IDIBAPS, Spain \\ Carlos Matute, Universidad del País \\ Vasco, Spain \\ *Correspondence: \\ Björn Rissiek, Department of \\ Neurology, University Medical \\ Center Hamburg-Eppendorf, \\ Martinistraße 52, 20246 Hamburg, \\ Germany \\ e-mail: b.rissiek@uke.de
}

Nanobodies are single domain antibodies derived from llama heavy-chain only antibodies (HCAbs). They represent a new generation of biologicals with unique properties: nanobodies show excellent tissue distribution, high temperature and $\mathrm{pH}$ stability, are easy to produce recombinantly and can readily be converted into different formats such as Fc-fusion proteins or hetero-dimers. Moreover, nanobodies have the unique ability to bind molecular clefts, such as the active site of enzymes, thereby interfering with the function of the target protein. Over the last decade, numerous nanobodies have been developed against proteins involved in inflammation with the aim to modulate their immune functions. Here, we give an overview about recently developed nanobodies that target immunological pathways linked to neuroinflammation. Furthermore, we highlight strategies to modify nanobodies so that they can overcome the blood brain barrier and serve as highly specific therapeutics for acute inflammatory brain injury.

Keywords: nanobodies, single domain antibodies, VHH, blood-brain barrier, neuroinflammation

\section{FROM LLAMA HEAVY-CHAIN-ONLY ANTIBODIES TO SINGLE DOMAIN NANOBODIES}

Mammalian immunoglobulins are composed of two heavy and two light chains which together form the antigen-binding paratope. In 1993, the group of Raymond Hamers demonstrated the existence of a new type of immunoglobulin in the serum of camels (Camelus dromedarius). These antibodies consist of heavy-chain dimers devoid of light chains, which brought them the name "heavy-chain-only antibodies" (HCAbs; HamersCasterman et al., 1993). These HCAbs are present in all members of the camelid family and account for 30 to $75 \%$ of circulating immunoglobulins (Hamers-Casterman et al., 1993; Sundberg and Mariuzza, 2002; Blanc et al., 2009; Muyldermans, 2013).

Structurally, the heavy-chains of HCAbs are composed of the antigen-binding variable domain $(\mathrm{VHH})$ followed by a hinge region and two constant domains $(\mathrm{CH} 2$ and $\mathrm{CH} 3)$, whereas the $\mathrm{CH} 1$ domain known from conventional antibodies is missing (Figure 1A; Hamers-Casterman et al., 1993). Apart from their unusual architecture, HCAbs also differ from conventional antibodies in their antigen recognition: VH and VL of conventional antibodies usually form a concave or flat shaped paratope suited for the binding of small molecules, peptides, or large antigens (Sundberg and Mariuzza, 2002; Blanc et al., 2009; Muyldermans, 2013). The paratope formed by a VHH domain, however, shows a convex shape and, therefore, enables the binding to molecular cavities or clefts, e.g., the active site of enzymes. Many enzymespecific VHH domains thereby act as antagonists (Figure 1B). This unique feature could be attributed to the long complementarity determining region 3 (CDR3) of the VHH domain which is able to form finger-like extensions (De Genst et al., 2006).

With approximately $15 \mathrm{kDa} \mathrm{VHHs}$ are the smallest naturally occurring antigen-binding protein domains. The name "nanobodies" was coined to reflect the small size of VHHs as recombinant proteins (Muyldermans, 2013). In order to generate nanobodies from HCAbs, llamas are immunized and boosted with the desired antigen. After the last boost, B cells are collected from peripheral blood to isolate mRNA, which is transcribed into cDNA. The gene region encoding for the VHH domain can be amplified via PCR and cloned into a phagemid vector. This strategy generates phages that express one particular nanobody clone on their surface and, at the same time, carry the DNA sequence encoding for this specific nanobody. Applying the phage display technology finally allows the selection of nanobody clones against the desired antigen (Clackson et al., 1991). Taken together, this approach allows the selection of target-specific nanobodies and, simultaneosly, delivers the DNA sequence coding for the selected nanobodies which then can be further used for recombinant expression (Wesolowski et al., 2009).

Most generated nanobodies are stable at high temperatures, low and high $\mathrm{pH}$, and other stringent conditions (Arbabi Ghahroudi et al., 1997; Dumoulin et al., 2002). Additionally, phage display selection can be performed under harsh conditions, e.g., the presence of detergents to improve the selection of more resistant clones. If applied in vivo, nanobodies display low toxicity and immunogenicity due to their small size, their relatively high sequence identity to human $\mathrm{VH}$, and to their rapid clearance from the periphery via the kidney (Hamers-Casterman et al., 1993; Muyldermans, 2013). To increase the in vivo half-life 
A
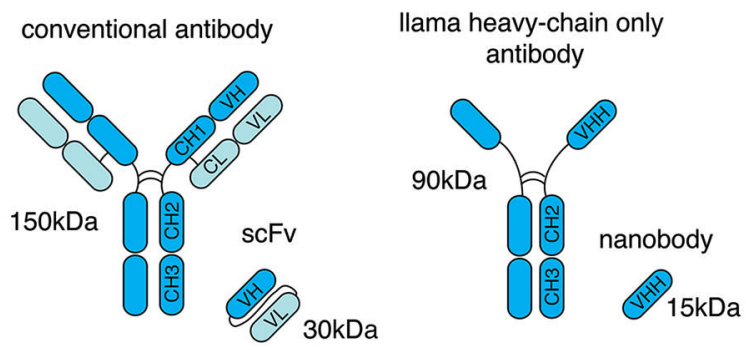

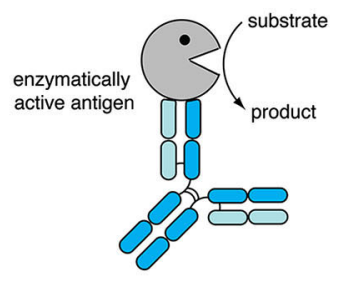

binding of planar epitopes

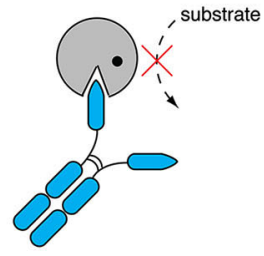

binding of cleft epitopes

C

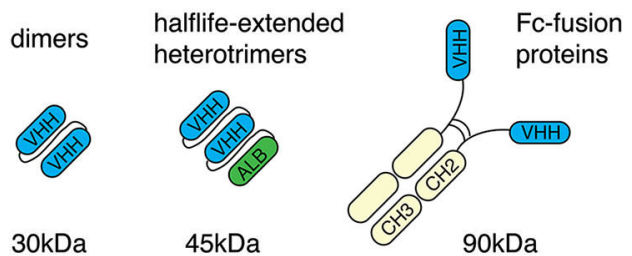

D

\begin{tabular}{|lllc|}
\hline Disease setting & Nanobody & Target & Phase \\
Inflammatory diseases & ALX-0761 & IL-17A/F & 1 \\
Rheumatoid arthritis & ALX-0061 & IL-6R & 2 \\
Rheumatoid arthritis & Ozoralizumab & TNFa & 2 \\
\hline Source: Ablynx & & & \\
\hline
\end{tabular}

FIGURE 1 | Nanobodies are single-domain antibodies derived from Ilama heavy-chain-only antibodies. (A) Conventional antibodies from mammals are composed of two heavy- and two light chains, camelidae additionally express antibodies devoid of ligh chains, so called heavy-chain only antibodies. (B) Heavy-chain-only antibodies can bind to molecular crevices thereby blocking the active site of enzymes. (C) Nanobodies derived from heavy-chain-only antibodies can be engineered as dimers, half-life-extended heterotrimers containing an anti-albumin nanobody or as dimeric Fc-fusionprotein. (D) Anti-inflammatory nanobodies are currently evaluated in clinical trials. nanobodies can be reformatted (converted into other formats by genetic engineering), e.g., to homodimers, heterotrimers containing an anti-serum-albumin nanobody (Sundberg and Mariuzza, 2002; Coppieters et al., 2006; Tijink et al., 2008; Blanc et al., 2009; Muyldermans, 2013) or nanobody-Fc-fusion proteins (Figure 1C). Thereby, nanobodies can be tailored for the desired in vivo application, e.g., small monomers for short-term in vivo imaging or half life-extended nanobodies for long-term therapeutic treatment (Hamers-Casterman et al., 1993; HassanzadehGhassabeh et al., 2013). The potential to antagonize targeted antigens, the high stability, the low toxicity and the possibility to tailor them for in vivo applications makes nanobodies a promising new generation of therapeutic proteins. To date, several antiinflammatory nanobodies are in clinical trials (Figure 1D), and more than 700 persons have received nanobodies in clinical trials without any adverse off-target side effects (Van Bockstaele et al., 2009; Williams, 2013).

\section{NANOBODIES AS MODULATORS OF IMMUNE CELLS AND INFLAMMATION}

In order to fight infectious diseases, numerous nanobodies have been generated against bacterial and viral antigens to prevent or ameliorate pathogenicity (Sundberg and Mariuzza, 2002; Blanc et al., 2009; Wesolowski et al., 2009; Muyldermans, 2013). More recently, key players of immunological pathways have come into focus as targets for nanobodies in order to modulate immune responses. This has resulted in the generation of nanobodies directed against Fc-receptors (FcR), chemokine receptors, chemokines, cytokines, and ecto-enzymes. These nanobodies often show high target specificities and are able to modulate the function of their target in an agonistic or antagonistic fashion.

\section{NANOBODIES DIRECTED AGAINST Fc-RECEPTORS}

FC receptors are expressed on the cell surface of diverse immune cells and are able to bind the Fc portion of antibodies thereby conducting either stimulatory or inhibitory signals, depending on the Fc receptor class (De Genst et al., 2006; Nimmerjahn and Ravetch, 2007). In 2008, Behar et al. described the isolation of Fc- $\gamma$-RIII-specific nanobodies from a llama immune library (Behar et al., 2008; Muyldermans, 2013). The selected nanobodies (C21 and C28) showed specific binding to both, Fc- $\gamma$-RIIIB and Fc- $\gamma$-RIIIA, and no binding to Fc- $\gamma$-RI or Fc- $\gamma$-RII. Binding of the Fc-part of an antibody to the Fc- $\gamma$-RIII on NK cells conducts an activating signal leading to the release of the proinflammatory cytokine interferon gamma (IFN $\gamma)$. Binding of nanobodies C2 1 and C28 in an agonistic fashion to Fc- $\gamma$-RIIIA on human NK cells induced the expression of IFN $\gamma$ (Clackson et al., 1991; Behar et al., 2008). In later studies, these nanobodies were used to generate Fab-like bispecific antibodies containing one nanobody directed against the Fc- $\gamma$-RIIIA and one directed against the carcinoembryogenic antigen (CEA; Behar et al., 2009; Wesolowski et al., 2009). By this strategy, the agonistic anti-Fc- $\gamma$-RIIIA nanobodies could be targeted to $\mathrm{CEA}^{+}$-tumor cells where they activate NK cells in situ inducing the lysis of the tumor cells. Further, injection of these bispecific constructs reduced the tumor growth in immunodeficient mice xenografted with $\mathrm{CEA}^{+}$-tumor cells when co-administered with human peripheral blood mononuclear cells (PBMCs; Arbabi Ghahroudi et al., 1997; Dumoulin et al., 2002; Rozan et al., 2013). It has to be evaluated whether FcR targeting 
nanobodies could also be applied as therapeutics for acute brain injury. A study using Fc- $\gamma$-R deficient mice showed a reduced infarct size compared to WT animals which could be linked to decreased microglia activation (Komine-Kobayashi et al., 2004).

\section{NANOBODIES DIRECTED AGAINST CHEMOKINE RECEPTORS AND CHEMOKINES}

The generation of functional monoclonal antibodies against Gprotein coupled receptors (GPCRs) such as chemokine receptors is notoriously difficult. With their unique binding features, nanobodies display a promising alternative for the generation of functional biologics to modulate chemokine receptor function, e.g., to inhibit immune cell migration to inflammatory sites. In 2010, the group of Martine Smit reported the generation of two nanobodies that specifically target the chemokine receptor CXCR4 (Jähnichen et al., 2010). Nanobodies 238D2 and 238D4 showed potent competitive inhibition of CXCL12 binding to CXCR4. When injected into monkeys, anti-CXCR4 nanobodies induced the mobilization of hematopoetic stem cells by disrupting the CXCR4/CXCL12 axis contributing to the residence of hematopoetic stem cells in the bone marrow. In 2013, the same group reported the generation of antagonistic nanobodies targeting CXCR7. Injected into mice, these nanobodies showed beneficial effects in an in vivo xenograft model of head and neck cancer (Maussang et al., 2013). Simultaneously, the same group published a panel of nanobodies specifically targeting CCL2, CCL5, CXCL11 and CXCL12. Binding of nanobodies to CXCL11 and CXCL12 inhibited chemokine receptor binding and thereby preventing chemokine receptor activation induced cell migration in vitro (Blanchetot et al., 2013). Since diverse chemokines and their receptors are known to contribute to the migration of immune cells to the brain after brain damage (Amantea et al., 2009), the nanobodies described above might be a promising therapeutic alternative for the treatment of acute brain injury.

\section{NANOBODIES DIRECTED AGAINST CYTOKINES}

Targeting and neutralization of proinflammatory cytokines by monoclonal antibodies is a promising strategy for the treatment of inflammatory diseases (Kopf et al., 2010). Tumor necrosis factor $\alpha(\mathrm{TNF} \alpha)$ was the first cytokine functionally targeted by nanobodies (TR2 = anti-human TNF $\alpha$, MT1 = anti-mouse $\mathrm{TNF} \alpha$ ). Expressed as bivalent molecules TR2-TR2 nanobodies showed a slightly higher neutralizing capacity than the TNF $\alpha$ neutralizing biologicals infliximab, adalimumab and etanercept (Coppieters et al., 2006). Further, heterotrimeric nanobodies (MT1-MT1-AR1) consisting of two MT1 and one serum albumin-binding nanobody (AR1) showed excellent therapeutic effects in the collagen-induced arthritis mouse model (Coppieters et al., 2006). Another study evaluating the therapeutic potential of bivalent $\mathrm{TNF} \alpha$ nanobodies in a mouse model of chronic colitis impressively demonstrated the versatility of the nanobody technology: genetically engineered Lactococcus lactis secreting MT1-MT1 bivalent anti-TNF $\alpha$ nanobodies profoundly reduced gut inflammation when daily administered by gavage (Vandenbroucke et al., 2010). Apart from neutralizing cytokines nanobodies can be used to "guide" cytokines to their desired target cells. In a proof-of-concept study Garcin et al. (2014) demonstrated that the toxicity of type I interferons, when applied in vivo, could be markedly reduced by genetically engineering fusion proteins of mutated IFN $\alpha 2$ with lower receptor affinities and nanobodies targeting programmed cell death 1 ligand 2 (PD-L2; Garcin et al., 2014). When injected into mice these fusion proteins preferentially induced IFN $\alpha 2$-mediated STAT1 phosphorylation in PD-L2 expressing cells in peritoneum and spleen, illustrating that nanobodies are valuable tools for "activity-by-targeting" based therapeutic approaches. For the treatment of acute brain injury, the nanobody-based neutralization of proinflammatory cytokines such as TNF $\alpha$ could be a promising approach to minimize inflammation-related further loss of brain tissue. Conversely, it is conceivable that nanobodies could be used to guide modified anti-inflammatory cytokines such as interleukin-10 to sites of brain inflammation to suppress inflammatory responses in situ.

\section{NANOBODIES DIRECTED AGAINST ECTO-ENZYMES}

The first cell surface resident ecto-enzyme targeted by nanobodies was murine ADP-ribosyltransferase C2 (ARTC2; Koch-Nolte et al., 2007; Menzel et al., 2013). ARTC2 is expressed on the cell surface of $\mathrm{T}$ cells and covalently attaches the ADP-ribose group of its substrate nicotinamide adenin dinucleotide (NAD) to arginine residues of several cell surface proteins. One wellcharacterized target of ARTC2 is the ATP-gated P2X7 ion channel. ADP-ribosylation of P2X7 on T cells induces channel opening and influx of calcium ions. Prolonged activation by ADP-ribosylation causes shedding of cell surface proteins such as CD62L and CD27, externalization of phosphatidylserin und ultimately cell death (Seman et al., 2003). Analyses of T cell subpopulations revealed different sensitivities to NAD-mediated cell death, with regulatory T cells (Tregs) and natural killer T cells (NKT cells) being highly susceptible (Hubert et al., 2010; Rissiek et al., 2014b). Antagonizing ARTC2 with nanobody s+16a prevents ADP-ribosylation of $\mathrm{P} 2 \mathrm{X} 7$ in vitro and in vivo. In a proof-of-principle study Scheuplein et al. showed that injection of $s+16 \mathrm{a}$ as $\mathrm{Fc}$-fusion protein restores an otherwise naturally NAD-depleted NKT cell population in diabetogenic NOD-CD38ko mice (Scheuplein et al., 2010). When activated in vivo by injection of $\alpha$-galactosylceramide, $s+16 a-$ restored NKT cells were capable of inhibiting the development of type 1 diabetes. A further study showed that injection of $s+16 a$ prevented ARTC2/P2X7 mediated cell death of highly susceptible Tregs and NKT cells during in vitro assays and adoptive transfer experiments, revealing the potential of $s+16 a$ as valuable tool for research and as potential therapeutic agent (Rissiek et al., 2014a). It has been shown that P2X7 activation is detrimental for the outcome of ischemic stroke (Arbeloa et al., 2012). Further, genetic deletion of the NAD-degrading ecto-enzyme CD38 in mice exacerbates ischemic damage (Choe et al., 2011), which might provide ARTC2 with an increased access to its substrate NAD. Therefore, s+16a could be used to clarify the role of the ARTC2/P2X7 axis during acute brain damage.

\section{MODIFYING NANOBODIES TO CROSS THE BLOOD-BRAIN BARRIER}

The therapeutic application of nanobodies has been tested in diverse mouse models of inflammation. However, therapeutic 
applications of biologics in neuroinflammatory diseases face an important biological barrier. The obstacle for effective delivery of therapeutic drugs, especially antibodies, is the blood brain barrier, which is only permeable for lipophilic molecules of up to $400 \mathrm{kDa}$ of size (Pardridge, 2012). The delivery of conventional antibodies to the brain is especially tedious because of Fc-receptor mediated efflux to the blood (Cooper et al., 2013). Therefore, nanobodies lacking an Fc-part represent a promising alternative to brain targeting monoclonal antibodies. Indeed, several groups have tested different strategies to deploy nanobodies as brain-drug deliverers or as bonafide brain-targeting drugs (Figure 2).

Muruganadam et al. described in 2002 the selection of a nanobody (FC5) that transmigrates across human blood-brainbarrier endothelium in vitro (Muruganandam et al., 2002). Later, the same group suggested that FC5 binds a putative $\alpha(2,3)$ sialoglycoprotein receptor and is transcytosed via clathrin vesicles (Abulrob et al., 2005). In a therapeutic experimental setup using the Hargreaves model of inflammatory pain, it was shown that FC5 conjugated with opioid peptide Dal could be deployed as drug delivery shuttle in vivo to induce a significant analgesic response in contrast to unconjugated Dal peptide (Farrington et al., 2014). Other approaches utilize receptor-mediated transcytosis for brain targeting. A recently published study showed that a fusionprotein of a peptide derived from apolipoprotein $\mathrm{E}$ (ApoE) and a model therapeutic protein ( $\alpha$-L-iduronidase) could be transferred to the brain via binding to the LDL receptor (LRP) expressed on cells of the blood-brain barrier (Wang et al., 2013). Furthermore, the transferrin receptor and the insulin receptor have also been exploited for receptor-mediated transcytosis of small molecule drugs and therapeutic proteins (Boado et al., 2012; Xiao and Gan, 2013). These studies indicate that nanobodies binding these receptors and triggering transcytosis could be a promising alternative to ligand-based delivery of drugs to the brain.

A study by Pierre Lafaye's group (Li et al., 2012) reported that nanobodies with a high isoelectric point (pI) spontaneously cross the blood brain barrier. Such nanobodies not only gained access to the brain but were even found to penetrate cells and bind to intracellular proteins. In a mouse study, the nanobody E9 ( $\mathrm{pI}=9.4)$ directed against glial fibrillary acidic protein (GFAP) crossed the $\mathrm{BBB}$ after injection via the tail vein and was able to bind to intracellularly expressed GFAP in astrocytes. Conjugation of fluorescent proteins to nanobody E9 (generating a "fluobody") allowed in vivo labeling of astrocytes, however, only if the basic pI was preserved. One possible limitation of this approach is that fairly large amounts $(2 \mathrm{mg}$ ) of nanobody had to be injected to obtain the desired effect. However, combining this approach-adjusting the pI to a basic level-with other approaches could possibly show beneficial effects. Indeed, the FC5 nanobody described above also has a basic pI (9.2) which might contribute to or facility transcytosis into the brain parenchyma

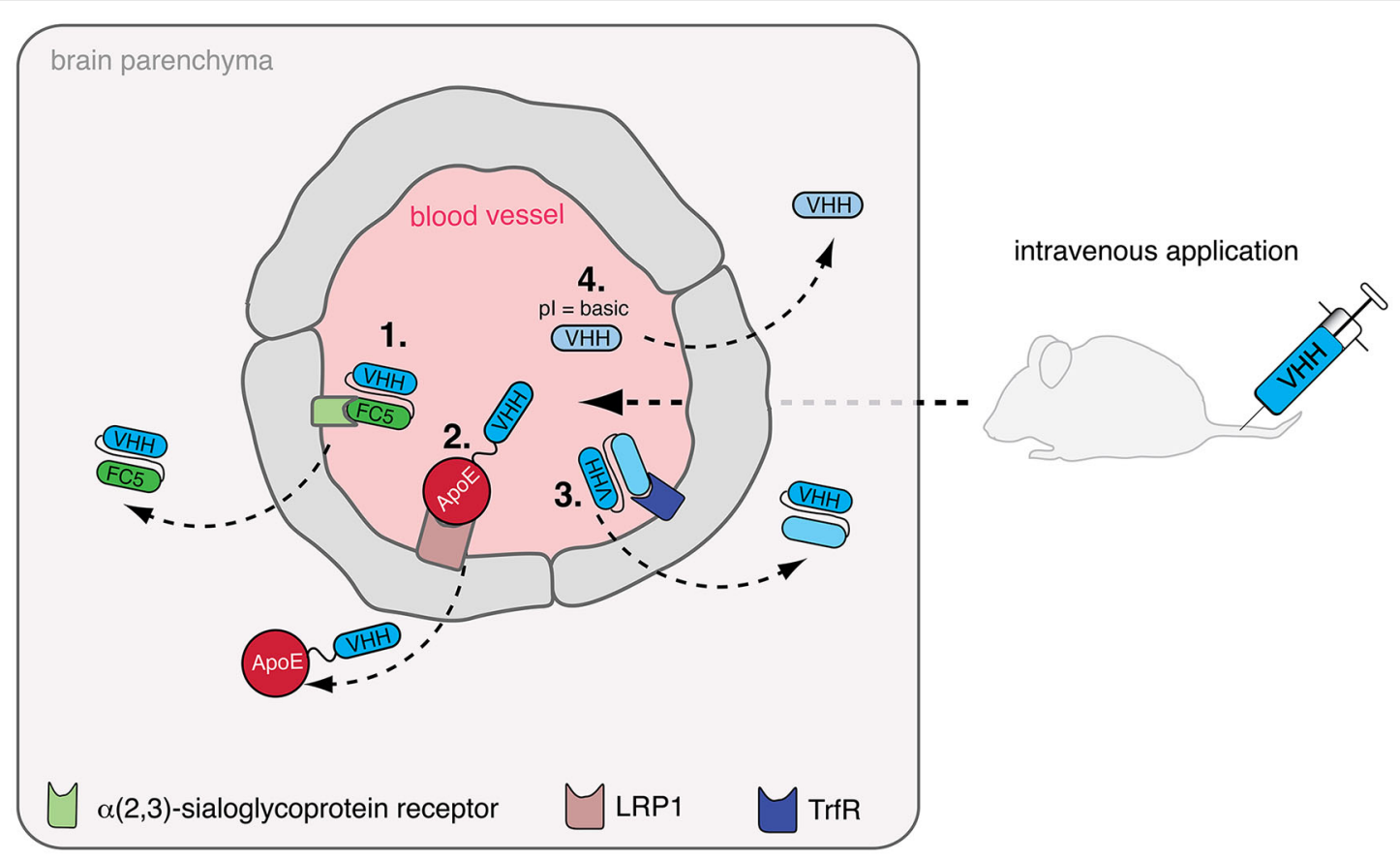

FIGURE 2 | Delivery of nanobodies to the brain. The blood-brain-barrier (BBB) hampers the delivery of intravenously injected nanobodies $(\mathrm{VHH})$ to the brain. To overcome this, diverse strategies are being developed: (1) Nanobody FC5, binding to a putative $\alpha(2,3)$-sialoglycoprotein receptor, can potentially be used as shuttling-nanobody to deliver other therapeutic proteins e.g., nanobodies to the brain. (2) Apolipoprotein $E(A p o E)$ binds to low density lipoprotein receptor-related protein 1 (LRP1) inducing transcytosis, which can be exploited as shuttle for therapeutic nanobodies. (3) In a similare fashion, other receptors triggering transcytosis across the BBB such as the transferrin receptor (TrfR) could be targeted for the transfer of therapeutic nanobodies. (4) Finally, shifting the isoelectric point ( $\mathrm{pl}$ ) of therapeutic nanobodies to a basic level facilitates crossing of the BBB by these nanobodies. 
(Farrington and Sisk, 2013). Further studies are needed to determine whether therapeutic nanobodies against inflammatory target proteins can be shuttled to the brain, e.g., by fusion to FC5 and by adjusting their $\mathrm{pI}$ with the aim of treating neuroinflammatory disease.

\section{IMPLICATION OF NANOBODIES TO TREAT ACUTE BRAIN INFLAMMATION}

After acute brain damage, e.g., ischemic stroke or trauma, the release of danger associated molecular pattern (DAMPs) from necrotic cells activates resident microglia, leading to the production of proinflammatory mediators such as cytokines and chemokines attracting other immune cells (Amantea et al., 2009; Iadecola and Anrather, 2011). Preventing local inflammation could be a means to prevent further loss of brain tissue. High mobility group box 1 (HMGB1) and nucleotide DAMPs such as adenosinetriphosphate (ATP) are released during cerebral ischemia (Magnus et al., 2012). Antibody-mediated neutralization of HMGB1 or antagonism of its receptor (receptor for advanced glycation end products, RAGE) markedly reduced the infarct size in a mouse ischemia/reperfusion model middle cerebral artery occlusion (MCAO; Muhammad et al., 2008). The ATP receptor $\mathrm{P} 2 \mathrm{X} 7$, which mediates inflammasome formation and cell death (Bartlett et al., 2014), was antagonized with small molecule inhibitors in a mouse model of transient focal ischemia, again resulting in a reduction of infarct size (Arbeloa et al., 2012). Both pathways, HMGB1/RAGE and ATP/P2X7, display promising targets for nanobody-mediated antagonism. However, release of DAMPs occurs shortly after the ischemic insult and before the breakdown of the BBB (Muhammad et al., 2008; CisnerosMejorado et al., 2014), requiring the generation of nanobodies that are able to cross the BBB applying the strategies described above. Currently, nanobodies directed against the $\mathrm{P} 2 \mathrm{X} 7$ receptor are under development (Laeremans et al., 2010).

An approach to control ischemia-related brain inflammation is to prevent migration of immune cells to the penumbra of ischemic lesions. Two independent studies demonstrated that blockade of the CXCL12/CXCR4 axis improved the functional outcome after stroke by attenuating post-ischemic inflammation (Huang et al., 2013; Ruscher et al., 2013). CXCL12-CXCR4 blockade was conducted using the small molecule inhibitor AMD3100. Since nanobodies against CXCL12 and CXCR4 have been generated (Jähnichen et al., 2010; Maussang et al., 2013), it may be worthwhile to evaluate their potential in ischemia/reperfusion animal models. To restrict the blockade of CXCR4 to infiltrating proinflammatory cells one could apply the "activity-by-targeting" strategy described above for IFN $\alpha 2$-guiding nanobodies. This could be useful in order to allow the CXCR4-dependend migration of cells important for brain recovery after stroke such as mesenchymal stem cells (Tsai et al., 2011). Furthermore, blockade of other chemokine receptors such as CXCR1 and CXCR2 by small molecule inhibitor Reparixin also reduces infiltration of proinflammatory neutrophiles and improves the motoric recovery after stroke (Sousa et al., 2013). Therefore, generation and application of nanobodies directed against CXCR1 and CXCR2 may represent one further strategy to ameliorate consequences of cerebral ischemia and beyond.

\section{CONCLUSIONS}

Nanobodies have been shown to be versatile and efficient biologicals suitable for therapy of inflammatory diseases. Due to their unique structure, nanobodies have the potential to modulate the function of cell surface and secreted proteins in an agonistic or antagonistic fashion. They can be genetically engineered to extend their half-life in vivo and, shown in a proof-ofconcept study, to serve as shuttles for the delivery of therapeutic agents across the BBB. Future studies will have to show whether this strategy could also be applied to deliver therapeutic nanobodies to the brain to ameliorate the consequences of neuroinflammation.

\section{ACKNOWLEDGMENTS}

We thank Dr. Anne Rissiek for critical reading of the manuscript. This work was supported by the ERA-NET NEURON research grant (NanoStroke) to Tim Magnus and Friedrich Koch-Nolte and a publication grant from NEUROWIND e.V. to Björn Rissiek.

\section{REFERENCES}

Abulrob, A., Sprong, H., Van Bergen en Henegouwen, P., and Stanimirovic, D. (2005). The blood-brain barrier transmigrating single domain antibody: mechanisms of transport and antigenic epitopes in human brain endothelial cells. J. Neurochem. 95, 1201-1214. doi: 10.1111/j.1471-4159.2005. 03463.x

Amantea, D., Nappi, G., Bernardi, G., Bagetta, G., and Corasaniti, M. T. (2009). Post-ischemic brain damage: pathophysiology and role of inflammatory mediators. FEBS J. 276, 13-26. doi: 10.1111/j.1742-4658.2008.06766.x

Arbabi Ghahroudi, M., Desmyter, A., Wyns, L., Hamers, R., and Muyldermans, S. (1997). Selection and identification of single domain antibody fragments from camel heavy-chain antibodies. FEBS Lett. 414, 521-526. doi: 10.1016/s00145793(97)01062-4

Arbeloa, J., Pérez-Samartín, A., Gottlieb, M., and Matute, C. (2012). P2X7 receptor blockade prevents ATP excitotoxicity in neurons and reduces brain damage after ischemia. Neurobiol. Dis. 45, 954-961. doi: 10.1016/j.nbd.2011. 12.014

Bartlett, R., Stokes, L., and Sluyter, R. (2014). The P2X7 receptor channel: recent developments and the use of P2X7 antagonists in models of disease. Pharmacol. Rev. 66, 638-675. doi: 10.1124/pr.113.008003

Behar, G., Chames, P., Teulon, I., Cornillon, A., Alshoukr, F., Roquet, F., et al. (2009). Llama single-domain antibodies directed against nonconventional epitopes of tumor-associated carcinoembryonic antigen absent from nonspecific cross-reacting antigen. FEBS J. 276, 3881-3893. doi: 10.1111/j.1742-4658.2009. 07101.x

Behar, G., Sibéril, S., Groulet, A., Chames, P., Pugnière, M., Boix, C., et al. (2008). Isolation and characterization of anti-FcgammaRIII (CD16) llama single-domain antibodies that activate natural killer cells. Protein Eng. Des. Sel. 21, 1-10. doi: 10.1093/protein/gzm064

Blanc, M. R., Anouassi, A., Abed, M. A., Tsikis, G., Canepa, S., Labas, V., et al. (2009). A one-step exclusion-binding procedure for the purification of functional heavy-chain and mammalian-type $\gamma$-globulins from camelid sera. Biotechnol. Appl. Biochem. 54, 207-212. doi: 10.1042/ba20090208

Blanchetot, C., Verzijl, D., Mujić-Delić, A., Bosch, L., Rem, L., Leurs, R., et al. (2013). Neutralizing nanobodies targeting diverse chemokines effectively inhibit chemokine function. J. Biol. Chem. 288, 25173-25182. doi: 10.1074/jbc.m113. 467969

Boado, R. J., Hui, E. K.-W., Lu, J. Z., and Pardridge, W. M. (2012). Glycemic control and chronic dosing of rhesus monkeys with a fusion protein of iduronidase and a monoclonal antibody against the human insulin receptor. Drug Metab. Dispos. 40, 2021-2025. doi: 10.1124/dmd.112.046375

Choe, C.-U., Lardong, K., Gelderblom, M., Ludewig, P., Leypoldt, F., Koch-Nolte, F., et al. (2011). CD38 exacerbates focal cytokine production, postischemic inflammation and brain injury after focal cerebral ischemia. PLoS One 6:e19046. doi: 10.1371/journal.pone.0019046 
Cisneros-Mejorado, A., Pérez-Samartín, A., Gottlieb, M., and Matute, C. (2014). ATP signaling in brain: release, excitotoxicity and potential therapeutic targets. Cell. Mol. Neurobiol. doi: 10.1007/s10571-014-0092-3. [Epub ahead of print].

Clackson, T., Hoogenboom, H. R., Griffiths, A. D., and Winter, G. (1991). Making antibody fragments using phage display libraries. Nature 352, 624-628. doi: 10. 1038/352624a0

Cooper, P. R., Ciambrone, G. J., Kliwinski, C. M., Maze, E., Johnson, L., Li, Q., et al. (2013). Efflux of monoclonal antibodies from rat brain by neonatal Fc receptor, FcRn. Brain Res. 1534, 13-21. doi: 10.1016/j.brainres.2013.08.035

Coppieters, K., Dreier, T., Silence, K., de Haard, H., Lauwereys, M., Casteels, P., et al. (2006). Formatted anti-tumor necrosis factor alpha VHH proteins derived from camelids show superior potency and targeting to inflamed joints in a murine model of collagen-induced arthritis. Arthritis Rheum. 54, 1856-1866. doi: 10. 1002/art.21827

De Genst, E., Silence, K., Decanniere, K., Conrath, K., Loris, R., Kinne, J., et al. (2006). Molecular basis for the preferential cleft recognition by dromedary heavy-chain antibodies. Proc. Natl. Acad. Sci. U S A 103, 4586-4591. doi: 10. 1073/pnas.0505379103

Dumoulin, M., Conrath, K., Van Meirhaeghe, A., Meersman, F., Heremans, K., Frenken, L. G. J., et al. (2002). Single-domain antibody fragments with high conformational stability. Protein Sci. 11, 500-515. doi: 10.1110/ps.34602

Farrington, G. K., Caram-Salas, N., Haqqani, A. S., Brunette, E., Eldredge, J., Pepinsky, B., et al. (2014). A novel platform for engineering blood-brain barriercrossing bispecific biologics. FASEB J. doi: 10.1096/fj.14-253369. [Epub ahead of print].

Farrington, G. K., and Sisk, W. (2013). Enhancement of transport of therapeutic molecules across the blood brain barrier. Patent

Garcin, G., Paul, F., Staufenbiel, M., Bordat, Y., Van der Heyden, J., Wilmes, S., et al. (2014). High efficiency cell-specific targeting of cytokine activity. Nat. Commun. 5:3016. doi: 10.1038/ncomms4016

Hamers-Casterman, C., Atarhouch, T., Muyldermans, S., Robinson, G., Hamers, C., Songa, E. B., et al. (1993). Naturally occurring antibodies devoid of light chains. Nature 363, 446-448. doi: 10.1038/363446a0

Hassanzadeh-Ghassabeh, G., Devoogdt, N., De Pauw, P., Vincke, C., and Muyldermans, S. (2013). Nanobodies and their potential applications. Nanomedicine (Lond) 8, 1013-1026. doi: 10.2217/nnm.13.86

Huang, J., Li, Y., Tang, Y., Tang, G., Yang, G.-Y., and Wang, Y. (2013). CXCR4 antagonist AMD3100 protects blood-brain barrier integrity and reduces inflammatory response after focal ischemia in mice. Stroke 44, 190-197. doi: 10. 1161/strokeaha.112.670299

Hubert, S., Rissiek, B., Klages, K., Huehn, J., Sparwasser, T., Haag, F., et al. (2010). Extracellular NAD+ shapes the Foxp3+ regulatory T cell compartment through the ART2-P2X7 pathway. J. Exp. Med. 207, 2561-2568. doi: 10.1084/jem. 20091154

Iadecola, C., and Anrather, J. (2011). The immunology of stroke: from mechanisms to translation. Nat. Med. 17, 796-808. doi: 10.1038/nm.2399

Jähnichen, S., Blanchetot, C., Maussang, D., Gonzalez-Pajuelo, M., Chow, K. Y., Bosch, L., et al. (2010). CXCR4 nanobodies (VHH-based single variable domains) potently inhibit chemotaxis and HIV-1 replication and mobilize stem cells. Proc. Natl. Acad. Sci. U S A 107, 20565-20570. doi: 10.1073/pnas. 1012865107

Koch-Nolte, F., Reyelt, J., Schössow, B., Schwarz, N., Scheuplein, F., Rothenburg, S., et al. (2007). Single domain antibodies from llama effectively and specifically block T cell ecto-ADP-ribosyltransferase ART2.2 in vivo. FASEB J. 21, 34903498. doi: 10.1096/fj.07-8661 com

Komine-Kobayashi, M., Chou, N., Mochizuki, H., Nakao, A., Mizuno, Y., and Urabe, T. (2004). Dual role of Fcgamma receptor in transient focal cerebral ischemia in mice. Stroke 35, 958-963. doi: 10.1161/01.str.0000120321.30 $916.8 \mathrm{e}$

Kopf, M., Bachmann, M. F., and Marsland, B. J. (2010). Averting inflammation by targeting the cytokine environment. Nat. Rev. Drug Discov. 9, 703-718. doi: 10. $1038 /$ nrd2805

Laeremans, T., Stortelers, C., Koch-Nolte, F., Gonzalez, M., Assuncao, J., and van Rompaey, P. (2010). Genetic immunization for producing immunoglobulins against cell-associated antigens such as $\mathrm{p} 2 \mathrm{x} 7, \mathrm{cxcr} 7$ or cxcr4. Patent

Li, T., Bourgeois, J. P., Celli, S., Glacial, F., Le Sourd, A. M., Mecheri, S., et al. (2012). Cell-penetrating anti-GFAP VHH and corresponding fluorescent fusion protein VHH-GFP spontaneously cross the blood-brain barrier and specifically recognize astrocytes: application to brain imaging. FASEB J. 26, 3969-3979. doi: 10.1096/fj.11-201384

Magnus, T., Wiendl, H., and Kleinschnitz, C. (2012). Immune mechanisms of stroke. Curr. Opin. Neurol. 25, 334-340. doi: 10.1097/WCO.0b013e32835 2ede6

Maussang, D., Mujić-Delić, A., Descamps, F. J., Stortelers, C., Vanlandschoot, P., Stigter-van Walsum, M., et al. (2013). Llama-derived single variable domains (nanobodies) directed against chemokine receptor CXCR7 reduce head and neck cancer cell growth in vivo. J. Biol. Chem. 288, 29562-29572. doi: 10. 1074/jbc.m113.498436

Menzel, S., Rissiek, B., Haag, F., Goldbaum, F. A., and Koch-Nolte, F. (2013). The art of blocking ADP-ribosyltransferases (ARTs): nanobodies as experimental and therapeutic tools to block mammalian and toxin ARTs. FEBS J. 280, 3543-3550. doi: $10.1111 /$ febs.12313

Muhammad, S., Barakat, W., Stoyanov, S., Murikinati, S., Yang, H., Tracey, K. J., et al. (2008). The HMGB1 receptor RAGE mediates ischemic brain damage. J. Neurosci. 28, 12023-12031. doi: 10.1523/JNEUROSCI.2435-08. 2008

Muruganandam, A., Tanha, J., Narang, S., and Stanimirovic, D. (2002). Selection of phage-displayed llama single-domain antibodies that transmigrate across human blood-brain barrier endothelium. FASEB J. 16, 240-242. doi: 10.1096/ fj.01-0343fje

Muyldermans, S. (2013). Nanobodies: natural single-domain antibodies. Annu. Rev. Biochem. 82, 775-797. doi: 10.1146/annurev-biochem-063011-092449

Nimmerjahn, F., and Ravetch, J. V. (2007). Fc-receptors as regulators of immunity. Adv. Immunol. 96, 179-204. doi: 10.1016/s0065-2776(07)96005-8

Pardridge, W. M. (2012). Drug transport across the blood-brain barrier. J. Cereb. Blood Flow Metab. 32, 1959-1972. doi: 10.1038/jcbfm.2012.126

Rissiek, B., Danquah, W., Haag, F., and Koch-Nolte, F. (2014a). Technical Advance: a new cell preparation strategy that greatly improves the yield of vital and functional Tregs and NKT cells. J. Leukoc. Biol. 95, 543-549. doi: 10.1189/jlb. 0713407

Rissiek, B., Haag, F., Boyer, O., Koch-Nolte, F., and Adriouch, S. (2014b). ADPRibosylation of P2X7: a matter of life and death for regulatory T cells and natural killer T cells. Curr. Top. Microbiol. Immunol. doi: 10.1007/82_2014_420. [Epub ahead of print].

Rozan, C., Cornillon, A., Pétiard, C., Chartier, M., Behar, G., Boix, C., et al. (2013). Single-domain antibody-based and linker-free bispecific antibodies targeting Fc $\gamma$ RIII induce potent antitumor activity without recruiting regulatory T cells. Mol. Cancer Ther. 12, 1481-1491. doi: 10.1158/1535-7163.mct12-1012

Ruscher, K., Kuric, E., Liu, Y., Walter, H. L., Issazadeh-Navikas, S., Englund, E., et al. (2013). Inhibition of CXCL12 signaling attenuates the postischemic immune response and improves functional recovery after stroke. J. Cereb. Blood Flow Metab. 33, 1225-1234. doi: 10.1038/jcbfm.2013.71

Scheuplein, F., Rissiek, B., Driver, J. P., Chen, Y.-G., Koch-Nolte, F., and Serreze, D. V. (2010). A recombinant heavy chain antibody approach blocks ART2 mediated deletion of an iNKT cell population that upon activation inhibits autoimmune diabetes. J. Autoimmun. 34, 145-154. doi: 10.1016/j.jaut.2009. 08.012

Seman, M., Adriouch, S., Scheuplein, F., Krebs, C., Freese, D., Glowacki, G., et al. (2003). NAD-induced T cell death: ADP-ribosylation of cell surface proteins by ART2 activates the cytolytic P2X7 purinoceptor. Immunity 19, 571-582. doi: 10. 1016/S1074-7613(03)00266-8

Sousa, L. F. D. C., Coelho, F. M., Rodrigues, D. H., Campos, A. C., Barcelos, L. D. S., Teixeira, M. M., et al. (2013). Blockade of CXCR1/2 chemokine receptors protects against brain damage in ischemic stroke in mice. Clinics (Sao Paulo) 68, 391-394. doi: 10.6061/clinics/2013(03)oa17

Sundberg, E. J., and Mariuzza, R. A. (2002). Molecular recognition in antibodyantigen complexes. Adv. Protein Chem. 61, 119-160. doi: 10.1016/s00653233(02)61004-6

Tijink, B. M., Laeremans, T., Budde, M., Stigter-van Walsum, M., Dreier, T., de Haard, H. J., et al. (2008). Improved tumor targeting of anti-epidermal growth factor receptor Nanobodies through albumin binding: taking advantage of modular Nanobody technology. Mol. Cancer Ther. 7, 2288-2297. doi: 10. 1158/1535-7163.mct-07-2384

Tsai, L.-K., Wang, Z., Munasinghe, J., Leng, Y., Leeds, P., and Chuang, D.-M. (2011). Mesenchymal stem cells primed with valproate and lithium robustly migrate to 
infarcted regions and facilitate recovery in a stroke model. Stroke 42, 2932-2939. doi: $10.1161 /$ strokeaha. 110.612788

Van Bockstaele, F., Holz, J. B., and Revets, H. (2009). The development of nanobodies for therapeutic applications. Curr. Opin. Investig. Drugs 10, 1212-1224.

Vandenbroucke, K., de Haard, H., Beirnaert, E., Dreier, T., Lauwereys, M., Huyck, L., et al. (2010). Orally administered L. lactis secreting an anti-TNF Nanobody demonstrate efficacy in chronic colitis. Mucosal Immunol. 3, 49-56. doi: 10. 1038/mi.2009.116

Wang, D., El-Amouri, S. S., Dai, M., Kuan, C.-Y., Hui, D. Y., Brady, R. O., et al. (2013). Engineering a lysosomal enzyme with a derivative of receptor-binding domain of apoE enables delivery across the blood-brain barrier. Proc. Natl. Acad. Sci. U S A 110, 2999-3004. doi: 10.1073/pnas.1222742110

Wesolowski, J., Alzogaray, V., Reyelt, J., Unger, M., Juarez, K., Urrutia, M., et al. (2009). Single domain antibodies: promising experimental and therapeutic tools in infection and immunity. Med. Microbiol. Immunol. 198, 157-174. doi: 10. 1007/s00430-009-0116-7

Williams, S. C. P. (2013). Small nanobody drugs win big backing from pharma. Nat. Med. 19, 1355-1356. doi: 10.1038/nm1113-1355
Xiao, G., and Gan, L.-S. (2013). Receptor-mediated endocytosis and brain delivery of therapeutic biologics. Int. J. Cell Biol. 2013:703545. doi: 10.1155/2013/703545

Conflict of Interest Statement: The authors declare that the research was conducted in the absence of any commercial or financial relationships that could be construed as a potential conflict of interest.

Received: 07 September 2014; paper pending published: 25 September 2014; accepted: 06 October 2014; published online: 21 October 2014.

Citation: Rissiek B, Koch-Nolte F and Magnus T (2014) Nanobodies as modulators of inflammation: potential applications for acute brain injury. Front. Cell. Neurosci. 8:344. doi: 10.3389/fncel.2014.00344

This article was submitted to the journal Frontiers in Cellular Neuroscience.

Copyright $\odot 2014$ Rissiek, Koch-Nolte and Magnus. This is an open-access article distributed under the terms of the Creative Commons Attribution License (CC BY).

The use, distribution and reproduction in other forums is permitted, provided the original author(s) or licensor are credited and that the original publication in this journal is cited, in accordance with accepted academic practice. No use, distribution or reproduction is permitted which does not comply with these terms. 